\title{
Dynamical modeling of the floral transition in legumes
}

\author{
Pavlinova P.A. ${ }^{1}$, Gursky V.V. ${ }^{1,2}$, Kozlov K.N. ${ }^{1}$, Samsonova M.G. ${ }^{1 *}$ \\ ${ }^{1}$ Peter the Great St. Petersburg Polytechnic University, St. Petersburg, Russia \\ ${ }^{2}$ Ioffe Institute, St. Petersburg, Russia \\ *e-mail:m.samsonova@spbstu.ru
}

Key words: flowering gene network, FLOWERING LOCUS T, chickpea, pea

Flowering time is an important stage of plant development, representing a transition from the vegetative to reproductive phases. Understanding processes controlling transition to flowering is significant for plant selection. The protein FLOWERING LOCUS T (FT) provides the key flowering signal mediating floral transition in the model plant Arabidopsis thaliana. This transcription factor is expressed in leaves and transfers to apical meristem of the plant, where it activates expression of the floral meristem identity genes $L E A F Y$ and APETALA1. In legumes, multiple $F T$ duplication occurred early in evolution, so that all legumes most probably have $F T$ orthologs from the following three clades: FTa, FTb, and FTc. This raises a question about the organization of the mobile flowering signals in legumes. We developed a dynamical model of the core gene network controlling transition to flowering and applied it to the previously published expression data for two closely related legume species, pea (Pisum sativum) and chickpea (Cicer arietinum, the early flowering ICCV 96029 cultivar). Both species have five FT-like genes, but the data-driven modeling results predict drastically different flowering signal organizations in pea and in the studied chickpea cultivar. For ICCV 96029, the model shows evidence that the inputs from different FT-like genes combine additively in the activation of flowering [1]. The pea orthologs of $F T$ have essentially different expression profiles, which resulted in a hypothesis about more complicated functional roles of $F T$ like genes in the flowering gene network in pea $[2,3]$. Our model confirms this hypothesis at the quantitative level. The comparative analysis of the regulatory architectures driving floral transition in pea and chickpea provides a first step in elucidating evolution of this regulatory module in legumes.

Acknowledgements: The study was supported by the Russian Foundation for Basic Research, grant 18-29-13033.

\section{References}

1. Gursky V.V., Kozlov K.N., Nuzhdin S.V., Samsonova M.G. Dynamical modeling of the core gene network controlling flowering suggests cumulative activation from the FLOWERING LOCUS T gene homologs in chickpea. Front. Genet. 2018;9:547.

2. Hecht V., Laurie R.E., Vander Schoor J.K. et al. The Pea GIGAS Gene Is a FLOWERING LOCUS $\mathrm{T}$ homolog necessary for graft-transmissible specification of flowering but not for responsiveness to photoperiod. Plant Cell. 2011;23:147.

3. Sussmilch F.C., Berbel A., Hecht V., Vander Schoor J.K., Ferrándiz C., Madueño F., Weller J.L. Pea VEGETATIVE2 is an FD homolog that is essential for flowering and compound inflorescence development. Plant Cell. 2015 Apr;27(4):1046-1060. 